\title{
Ernest Henley's Isospin and the Ensuing Progress
}

\author{
G.A. Miller \\ Department of Physics, University of Washington, Seattle, WA 98195-1560
}

(Dated: October 15, 2018)

\begin{abstract}
Ernest Henley's contributions to understanding isospin symmetry and the experimental and theoretical progress that followed are reviewed. Many experimentalists and theorists worked to bring Ernest's early vision of the subject to fruition. This progress and wide-ranging impact is described.
\end{abstract}
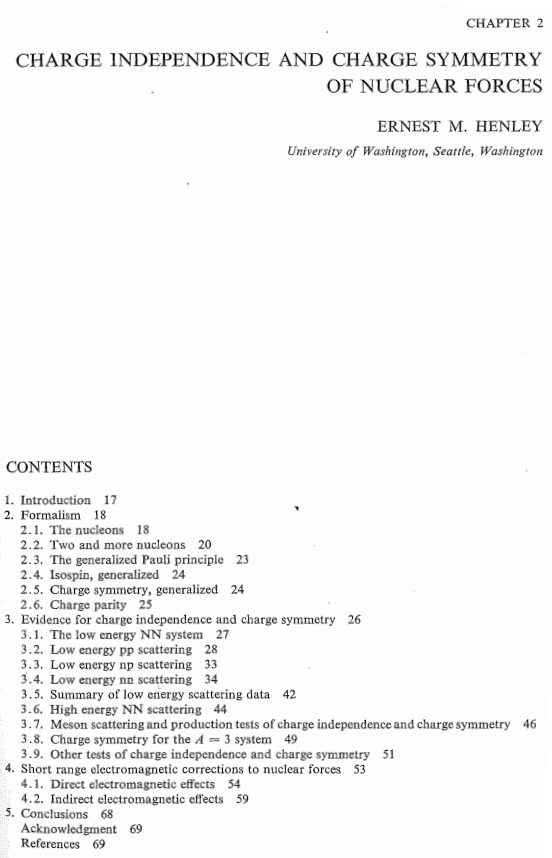

FIG. 1: First page of Ernest Henley's 1970 article

I was very happy to contribute to this volume intended to honor the contributions of Ernest Henley, a man who had a very major influence on my career. My first encounter with Ernest occurred about two years before I met him. I was a graduate student at MIT, studying isospin violating hadronic corrections to the widths of isobaric analog states, working with Arthur Kerman. Some time around 1970, John Negele told me that there was a new preprint on isospin violations from Henley [1] in the Physics Library. The article was much more advanced than any on that topic that I had ever seen. I thought: What is a man like that doing in Seattle? I was soon to find out!

I think that it is fair to say that Ernest established the field of isospin as a fundamental symmetry. The first page of the article, shown in Fig. 1, displays the scope of his work. Note that it was Chapter 2, the first real chapter after the Introduction, in a 750 page book of 14 chapters. This paper established isospin as an approximate symmetry, collected the evidence for this statement, and originated various terms. Indeed Ernest coined the term "approximate symmetry" to denote a symmetry that was slightly broken by small effects.

Ernest organized the nuclear force. It is a definite fact that the neutron-proton (np) and nn nuclear forces are different, but these forces were called charge independent! Ernest defined charge independence as a symmetry in which the $p p, n p$ and $n n$ forces are equal to each other in the same space-spin state. For nuclear forces, charge symmetry was defined as the identity of $n n$ and $p p$ forces. Ernest generalized these statements to all hadrons. 
Electromagnetic forces break these symmetries, as expected. Ernest also catalogued the non-electromagnetic forces.

One detail that proved very important involves the difference between isospin invariance (charge independence) and charge symmetry. Isospin invariance means that the physics is independent of any rotation in isospin space. For this to be true one needs $\left[H, T_{i}\right]=0$, where $T_{i}$ is a component of the isospin operator. Charge symmetry is invariance under a particular rotation: by $180^{\circ}$ about the $y$-axis, if the $z$-axis is associated with charge:

$$
P_{\mathrm{cs}} \equiv e^{i \pi T_{2}}
$$

Charge symmetry does not imply isospin invariance. The breaking of charge symmetry does imply the breaking of charge independence.

Ernest defined future directions for the field. Regarding theory, he wrote "Unless an understanding of the hadronic NN force is possible, it will remain extremely difficult to make convincing theoretical calculations of non-electromagnetic hadron-hadron interactions." For experiment he advocated further work to accurately establish the $n n$ scattering length. In particular, he focussed on the reaction $\pi^{-}+D \rightarrow n n+\gamma$. The theory was carried out brilliantly by Gibbs, Gibson \& Stephenson [2]. This was the first paper I saw that presented an error analysis of the theory. Later measurements of the reaction $[3,4]$ led to establishing the fact that the $n n$ interaction is more attractive than the $n p$ one in the ${ }^{1} S_{0}$ state.

\section{NEXT STEPS}

I arrived in Seattle in Sept. 1975. In the following January Ernest asked me to join in writing a review [5]. He said that charge independence breaking was well-established, therefore the focus should be on charge symmetry breaking, CSB. My memory is that Ernest had almost the complete outline written at the time he asked me to help. I supplied some technical details. The wide scope of the article is apparent from the first page, Fig. 2. Along with the review, many papers on this subject ultimately resulted. The earliest were Refs. [6-8]. I also eventually wrote further reviews [9-11].

Ernest's summary of the theory was interesting. He wrote that CSB was difficult to calculate, but at least it could stimulate experiment. For experiment he/we had three suggestions. One could test the center of mass polarization relation $P_{n}(\theta)=P_{p}(\pi-\theta)$. Charge symmetry would be broken if the cross section for $d d \rightarrow{ }^{4} \mathrm{He}+\pi^{0}$ reaction were measured to be non-vanishing. Another proposed test was the angular asymmetry about $90^{\circ}$ in the center of mass of the reaction $n p \rightarrow d \pi^{0}$.

Ernest was not content to merely publish papers. He actively worked with the committees that chose experiments at the TRIUMF and IUCF laboratories. I later worked with experimenters at those labs on related

theoretical issues. The experimental progress on these reactions is discussed below, but first it worthwhile to provide some detail regarding isospin breaking in the nucleon-nucleon system.

\section{HENLEY-MILLER NUCLEON-NUCLEON FORCE CLASSIFICATION (1977)}

We review the CSB and CIB terminology of nucleon-nucleon forces [5].

Class (I): Forces which are isospin independent that commute with all components of the isospin operator. Such forces, $V_{I}$ have an isoscalar form,

$$
V_{I}=a+b \vec{\tau}(i) \cdot \vec{\tau}(j)
$$

where $a$ and $b$ are Hermitian isospin independent operators and $i \neq j$.

Class (II): Forces which maintain charge symmetry, but break charge independence. These can be written in isostensor form,

$$
V_{I I}=c\left(\tau_{3}(i) \tau_{3}(j)-\vec{\tau}(i) \cdot \vec{\tau}(j)\right.
$$




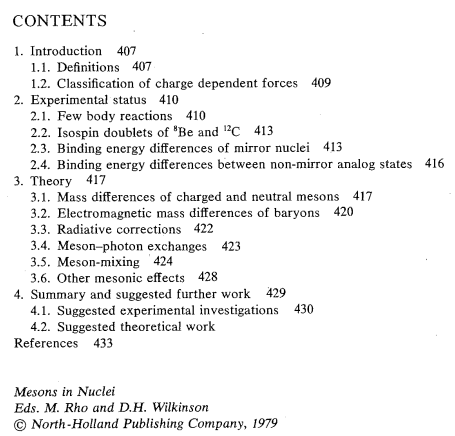

FIG. 2: First page of our 1977 article [5]

The Coulomb interaction leads to a Class II force as do the effects of the pion mass difference in pion exchange forces. Effects of charge-dependent coupling constants may also lead to such a Class II force.

Class (III): Forces which break both charge independence and charge symmetry, but which are symmetric under the interchange $i \leftrightarrow j$ in isospin space,

$$
V_{I I I}=d\left(\tau_{3}(i)+\tau_{3}(j)\right) .
$$

A Class III force differentiates between $n n$ and $p p$ systems, but does not cause isospin mixing in the two-nucleon system because

$$
\left[V_{I I I}, T^{2}\right]=0 .
$$

The effects of $\rho^{0}-\omega$ mixing yields such a force, as does the Coulomb interaction.

Class (IV) Class IV forces break charge symmetry and therefore charge dependence; they cause isospin mixing. These forces take the form

$$
V_{I V}=e(\sigma \overrightarrow{(i)}-\sigma(\vec{j})) \cdot \vec{L}\left(\tau_{3}(i)-\tau_{3}(j)\right)+f(\vec{\sigma}(i) \times \vec{\sigma}(j)) \cdot \vec{L}\left(\tau_{3}(i) \times \tau_{3}(j)\right),
$$

where $\vec{L}$ is the two-nucleon orbital angular momentum $e$ and $f$ are Hermitian isospin-independent operators. Such forces give CSB spin-orbit effects that account for the np analyzing power differences [12-17] and contribute to nuclear isospin mixing [18].

\section{SEARCH FOR CLASS IV FORCES}

Charge symmetry leads to the equality of the differential differential cross sections for polarized neutrons scattering from unpolarized protons and vice versa. As a result $A_{n}(\theta)=A_{p}(\theta)$ where $A_{n, p}$ denotes the analyzing power and where the subscript represents the polarized nucleon. (Time reversal invariance dictates 
that $A(\theta)=P(\theta)$.) A non-vanishing asymmetry difference is directly proportional to the isotopic spin singlettriplet, spin singlet-triplet mixing amplitude and therefore direct evidence of a Class IV force. The first measurement of charge symmetry breaking in $n p$ elastic scattering (at an incident neutron energy of 477 $\mathrm{MeV}$ ) was performed at TRIUMF $[12,13]$ by measuring the difference $A_{n}-A_{p}$, at the zero-crossing angle of the average analyzing power. A non-zero result, which was largely explained by the effect of the neutron-proton mass difference on the one-pion exchange potential [19, 20], was observed. This discovery was followed by confirmation by an accurate IUCF experiment $[16,17]$. Later TRIUMF work at $347 \mathrm{MeV}[14,15]$ also found a significant effect. In general, the measured analyzing power differences of the IUCF and TRIUMF experiments were well reproduced by theoretical predictions based on the paradigm at the time: meson exchange potential models, which indirectly incorporate quark level effects. The calculations include contributions from one photon exchange (the magnetic moment of the neutron interacting with the current of the proton), from the neutron-proton mass difference affecting charged single $\pi, \rho$ and $\omega$ exchange, and from isospin mixing $\rho^{0}-\omega$ meson exchange. We note that all of the model parameters related to the strong interaction were constrained fits to phase shifts, so that the calculations were testing only the inputs related to charge symmetry breaking. The review [10] discusses this topic in more detail.

\section{THE REACTION $d d \rightarrow \alpha \pi^{0}$}

Ernest Henley's words "if isospin is conserved this reaction is forbidden, however contrary to all claims made in the literature, this reaction only tests charge parity" are very interesting. Ernest was willing to call out his colleagues in general, but was not particularly disposed to publicly naming names. Charge parity was Ernest's phrase for charge symmetry.

A successful measurement of the reaction was made at IUCF [21] after several incomplete attempts at other laboratories. The authors reported the first observation of the charge symmetry breaking $d d \rightarrow \alpha \pi^{0}$ reaction near threshold. by measuring total cross sections for neutral pion production of $12.7 \pm 2.2 \mathrm{pb}$ at $228.5 \mathrm{MeV}$ and $15.1 \pm 3.1 \mathrm{pb}$ at $231.8 \mathrm{MeV}$. These cross sections arise fundamentally from the down-up quark mass difference and quark electromagnetic effects that contribute in part through meson mixing. These values of the cross section were qualitatively reproduced by theoretical calculations [22-25]. Note the small value of the measured cross sections. This arises because the cross section is amazingly the square of a charge symmetry breaking amplitude. All other measurements of charge symmetry breaking are essentially first-order effects.

The IUCF program was followed further observations at higher energies performed at the WASA-at-COSY facility $[26,27]$. The review [11] discusses this topic in detail.

\section{THE REACTION $n p \rightarrow d \pi^{0}$}

An asymmetry about $90^{\circ}$ in the center-of mass $(\mathrm{cm})$ frame in the reaction $n p \rightarrow d \pi^{0}$ can only be caused by charge symmetry breaking. This is shown in Fig. 3. The size of the charge symmetry breaking can be inferred from the cm $n p \rightarrow d \pi^{0}$ forward-backward asymmetry, $A_{\mathrm{fb}}$, defined as

$$
A_{\mathrm{fb}} \equiv \frac{\int_{0}^{\pi / 2} d \Omega(\sigma(\theta)-\sigma(\pi-\theta))}{\int_{0}^{\pi} d \Omega \sigma(\theta)},
$$

where $\theta$ is the $\mathrm{cm}$ angle between the incident neutron beam and the scattered deuteron. A successful measurement was made [28] in 2003. The review [11] discusses this topic in more detail.

\section{ISOSPIN IN QCD}

QCD was yet to be discovered at the time of Henley's 1970 review. The notion that quantum chromodynamics (QCD), with its quarks and gluons, is the fundamental theory of the strong interactions generated 

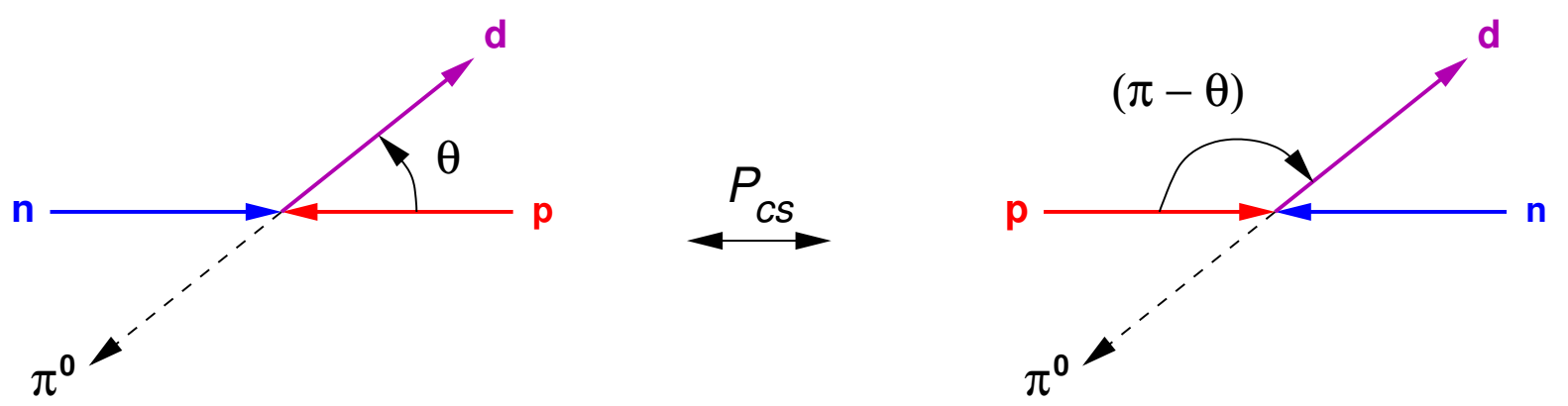

FIG. 3: Charge symmetric relations for the reaction $n p \rightarrow d \pi^{0}$ in the center of mass frame. The rotation $P_{c s}$ (Eq. (1)) rotates the neutron into a proton and vice versa

many new perspectives for studying hadronic systems and interactions. In QCD the interactions of up $(u)$ and down $(d)$ quarks are identical, if the $u d$ quark mass difference is neglected. This identity of interactions is termed charge symmetry. The definition of the charge symmetry operation $P_{\mathrm{cs}}$ is given in Eq. (1). In QCD $u$ and $d$ quarks carry the isospin quantum numbers and the charge symmetry operator changes $d$ into $u$ quarks, and $u$ into $d$ quarks [9]

$$
P_{\mathrm{cs}}|d\rangle=|u\rangle, P_{\mathrm{cs}}|u\rangle=-|d\rangle .
$$

In this language, the quark mass difference plus electromagnetic effects is responsible for charge symmetry breaking. It is also true that in this language, the quark mass difference plus electromagnetic effects is responsible for all of isospin breaking, so that the distinction between charge symmetry breaking and isospin breaking becomes less pronounced. Nevertheless, the distinction between charge symmetry breaking and isospin violation remains very important at the hadronic level. For example, the mass difference between the neutron and the proton is an example of charge symmetry breaking (CSB), but the $\pi^{ \pm}-\pi^{0}$ mass difference is not.

In QCD the only sources of CSB are electromagnetic interactions and the mass difference between up and down quarks, so CSB studies are necessarily concerned with the origins and consequences of the small quark mass difference. Therefore, the relationship between the fundamental quark-gluon dynamics and observable hadronic effects is a natural goal of the CSB studies discussed in previous sections. The QCD basis of CSB is discussed in detail in the review [9]. The consequences of the up-down quark mass difference, as elaborated via effective field theory are discussed in the review [11]. Some of that material is recounted in the next Section.

\section{ISOSPIN IN EFFECTIVE FIELD THEORY}

Effective field theory, EFT, is the modern technique to evaluate the consequences of QCD for hadronic and nuclear physics. This is possible because the most general Lagrangian which respects unitarity, has correct properties under cluster decomposition and the same symmetries as QCD, should be equivalent to QCD [29]. Thus one may use chiral hadronic Lagrangians, to make QCD predictions at relatively low energies [30-32]. Predictive power is retained when at low energies an expansion in momentum, formulated using power counting arguments, can be shown to converge.

The principal aim of EFT as applied to nuclear physics is to handle the short distance (or high momentum) physics, the region that most concerned Ernest Henley. One often resorts to using models to deal with shortdistance $(\approx 0.7 \mathrm{fm})$ interactions interactions between two or more nucleons. EFT offers a systematic procedure 
of treating ultraviolet-divergent integrals that appear in evaluating loop diagrams by using cutoffs at a scale $\Lambda$ and counter terms with magnitude expressed in terms of low energy constants LECs that depend implicitly on $\Lambda$. If the Lagrangian and renormalization procedure is correctly constructed, computed observables are independent of $\Lambda$. In making calculations one expands low-energy observables, that depend upon a momentum scale $Q$, in powers of the hopefully small parameter $Q / \Lambda \ll 1$ and argues that only a finite number of LECs contribute at a given order in the $Q / \Lambda$ expansion. Thus one hopes to obtain a Lagrangian with a small number of coefficients that can be determined from experimental data, fundamental theory, or models. The resulting Lagrangian can then be used to predict other observables.

To understand charge symmetry breaking in nuclear systems, the Lagrangian should be expressed in terms of pions, nucleons, $\Delta$ 's, and photons. Unitarity and correct cluster decomposition must be retained in any effective theory, but the essential feature of QCD is its approximate chiral symmetry (for small quark masses), which imposes important restrictions on the way pions interact. The quark mass difference breaks isospin explicitly. In low-energy EFT, the effect of quark-mass terms can be reproduced if all terms that break chiral symmetry in the same way are included. These interactions can involve the pionic fields without derivatives, but are always accompanied by powers of $m_{u}+m_{d}$ or $m_{u}-m_{d}$.

\section{EFT and pion production}

The production of a single pion in a nucleon-nucleon collision has a rather special role because of its relation with chiral symmetry. Furthermore, it is a source of $\pi^{0}$ that can access the potentially large effects of charge symmetry breaking [33] (see below). One might therefore hope that effective field theories will give insights and allow the determination of $m_{d}-m_{u}$.

Chiral symmetry provides the conditions for the construction of an effective field theory, in that it forces the mass of the pion $m_{\pi}$, as the Goldstone boson of the chiral symmetry breaking, to be low, and the interactions to be weak. In the chiral limit $\left(m_{\pi}=0\right)$ the pion must be be free of interactions for vanishing momenta.

\section{EFT and $C S B$}

The mass difference between $u$ and $d$ quarks breaks isospin symmetry explicitly. Indication from the meson masses is that the ratio $\varepsilon \equiv\left(m_{u}-m_{d}\right) /\left(m_{u}+m_{d}\right) \sim 1 / 3$. Naively, this suggests that isospin might not be a much better symmetry than the rest of the chiral group. On the other hand, a quick look at hadron masses and much experimental evidence show that isospin is typically broken only at the few percent level.

That isospin is such a good symmetry can be understood from the pattern of chiral-symmetry breaking incorporated in the chiral Lagrangian [34]. While explicit chiral-symmetry-breaking effects are present already at the lowest order in $Q / \Lambda$ through the pion mass term, operators generated by the quark mass difference appear only at the next order through a term that contributes to the nucleon mass splitting and, due to chiral symmetry, to certain pion-nucleon interactions. As a consequence, in most quantities isospin breaking competes with isospin-conserving operators of lower order, and its relative size is not $\varepsilon$ but $\varepsilon\left(Q / M_{Q C D}\right)^{n}$, where $n$ is a positive integer. In other words, isospin is an accidental symmetry [34]: a symmetry of the lowest order EFT which is not a symmetry of the underlying theory.

The only known exception to this rule is in the isoscalar $t$ channel in $\pi N$ scattering at threshold. In this case, there is no contribution from the leading-order Lagrangian, and both the isospin-conserving and -violating amplitudes start at the same order. The isospin-violating piece comes from the pion-nucleon interactions linked to the nucleon mass splitting. As described in previous sections, the effects have been seen experimentally, but many possible terms involving different manifestations of the up-down quark mass difference contribute to the scattering amplitude.

Note that the classes I-IV of a previous section have been shown to correspond to the increasing orders of chiral perturbation theory $[34,35]$. Class I is lowest order, Class II is of the next order, and so on. 


\section{CSB NUCLEON ELECTROMAGNETIC FORM FACTORS AND PARITY VIOLATING (PV) ELECTRON SCATTERING}

The basic idea is that elastic PV electron-proton scattering is sensitive to nucleon strangeness content [36],

and also to the value of the weak-mixing angle $[37,38]$. So far, a convincing signal for strangeness in the nucleon has not been seen [36].

The relevance of charge symmetry or its breaking to PV electron scattering on the proton arises from the need to relate the amplitude for $Z$-boson absorption on the proton to measured proton and neutron electromagnetic form factors. This can be done only if charge symmetry holds to an extent that its breaking is much smaller than any expected contribution from strangeness.

The breaking of charge symmetry brings in a correction that cannot be obtained directly from experimental observations [39-41]. The key question is whether the uncertainty in obtaining the correction is large compared to current and projected experimental uncertainties. Experimentalists stated that charge symmetry limits the ability to push further on the strange form factors because results obtained with improved precision would be hard to interpret cleanly in terms of strangeness or CSB.

I addressed [40] the question of whether or not CSB really limits the ability to push further. That paper found that the CSB corrections are less than $1 \%$ of the size of the electromagnetic form factors $G_{E}, G_{M}$. When re-expressed in terms of absolute values of charge symmetry breaking form factors, the results were very small of order $2 \times 10^{-3}$. This is small enough to ignore, despite the amazingly high accuracy of current experiments.

However, I had ignored the effect of charge symmetry breaking arising from the influence of the neutronproton mass difference on the pion cloud of the nucleon. This effect was included by Kubis \& Lewis [41]. These effects are not small because of a log divergence in the loop integrals. In their resonance-saturation procedure the pion graph is cut off at the mass of the rho meson, and rho-omega mixing graphs provide a finite counter term. This is larger than the pion diagrams. The result, the charge symmetry breaking magnetic form factor ranges between 0.01 and 0.04 , or about 10 times larger than my result. There is also a large uncertainty in the results due to lack of knowledge of the $\omega$ nucleon strong tensor coupling.

Kubis \& Lewis [41] take the strong coupling constants from dispersion analyses of electromagnetic form factors based on vector meson dominance. Such fits are well known to be flexible [42, 43]. The strong coupling constants for omega nucleon coupling are about seven times larger than used in NN scattering. So there is a conflict.

How can we tell which method (or if either method) is correct? One answer is that the effects of rhoomega mixing in nucleon-nucleon scattering is constrained. It is known to give a medium range class III CSB potential that can account for the scattering length difference between $n n$ and $p p$ systems [9, 44], and a class IV CSB potential that plays an important role in understanding CSB in np scattering. The class III potential may account for the missing binding energy difference between ${ }^{3} \mathrm{He}$ and ${ }^{3} \mathrm{H}[45]$ and also the Nolen-Schiffer anomaly [46], see the review [10]. There are other possible sources of CSB that influence these energy differences. All effects have the same sign because the are all driven by the mass difference between up and down quarks. The use of the KL coupling constants gives potentials that are rather different, and much larger, than the one [45] needed phenomenologically.

We [47] made later calculations of the CSB form factors using relativistic chiral perturbation theory. The use of relativistic chiral perturbation theory leads to finite and convergent results. The CSB effects were found to be an order of magnitude smaller than current experimental bounds on proton strangeness.

More generally, I wish to address a bias. I did a quark model calculation. Kubis \& Lewis did a chiral perturbation theory calculation. One usually thinks that a theory is better than a model. However, if an unconstrained counter term is needed to evaluate the theory, then a model that is constrained by experimental data is better than a theory.

\section{OTHER SUBJECTS}

I want to briefly mention three other topics that are relevant to more modern topics. 


\section{Super allowed Fermi $\beta$ decay}

In my opinion, the computations of the rate for nuclear super allowed beta decay, used to test the unitarity of the CKM matrix, could be improved. In particular, we [48, 49] used an exact formalism to show that certain radial excitations that are often neglected are significant. My impression is that it is difficult to include such effects in ab initio calculations. Nevertheless, given the importance of the subject, I hope that someone will look further at this.

\section{The NUTEV anomaly}

The NuTeV group[50] has measured charged and neutral current reactions for deep inelastic scattering of neutrinos by iron targets. Ratios of these cross sections can be used to determine the Weinberg angle, provided that the target is isoscalar, the nuclear strangeness content can be ignored, charge symmetry holds, and a variety of nuclear effects on deep inelastic scattering can be neglected. In this case the Paschos-Wolfenstein [51] relation holds and $\sin ^{2} \theta_{W}$ can be extracted from ratios of neutral current to charged current total cross sections, averaged with the proton and neutron. This simple relation follows from equating the $u, d$ contributions in the proton scattering with those of the $d, u$ contributions to the neutron scattering, and then taking the difference between the neutrino and anti-neutrino cross sections. This isolates the axial-vector interference term

proportional to $\frac{1}{2}-\sin ^{2} \theta_{W}$ for the neutral current. The $\mathrm{NuTeV}$ value for $\sin ^{2} \theta_{W}$ is three standard deviations larger than the value measured in other electroweak processes. However charge symmetry breaking causes a change in the Paschos-Wolfenstein relation [52-54], and this correction has been shown to be proportional to a ratio of quark momenta that is independent of $Q^{2}$ and essentially model-independent [55]. The size of the correction is about $50 \%$ of the discrepancy with other determinations of the Weinberg angle [55]. There are other nuclear and hadronic effects that complicate the interpretation of this reaction. See e.g. [56]

\section{$m_{d}-m_{u}>0$ CAUSES ALL STRONG-INTERACTION CHARGE SYMMETRY BREAKING}

There is a very definite striking pattern caused by the positive value of $m_{d}-m_{u}>0$. The neutron mass is greater than the proton mass, in contrast with the expectations based on electromagnetic effects. But this is not all, as detailed in Table 1.1 of Ref. [9]. The mass difference between the members of dozen hadronic isospin doublets may be understood by counting the number of up and down quarks. This pattern occurs for mesons and baryons. Moreover, this mass difference is responsible for the mixing of mesons. Electromagnetic effects also violate charge symmetry, but in most cases these are smaller than the strong-interaction effects of $m_{d}-m_{u}>0$.

A recent paper [57] uses a model in which "the Coulomb interaction is the source of all isospin breaking". The basic idea of this work is that at short center-of -mass separations between two nucleons "the six quarks intermixed give more possibilities for correlations among them leading to additional Coulomb-energy." This may be true, but the neglect of the effects of the quark mass difference is a very significant omission. Such give rise to significant effects in nucleon-nucleon scattering and to mass differences between mirror nuclei [58]. In particular that reference found that "the computed mass difference between six quark clusters formed by two neutrons and two protons is less than that $(2.6 \mathrm{MeV})$ of free nucleons in both the non-relativistic and MIT bag quark models. This is because quark-quark Coulomb and hyperfine interactions have an increased magnitude in the six-quark system. This makes a neutron-rich nucleus slightly more bound than its protonrich mirror. The magnitude of the effect is of about the right size to account for the missing Coulomb energy problem (Nolen-Schiffer anomaly) for nuclei with $\mathrm{A}=3,13,17,29,33$, and 41." The six-quark clusters have very different structure than two-nucleons, being mainly hidden color configurations. That paper also shows that the Coulomb effects give attraction, but the effects of the light quark mass difference on the kinetic energy and the hyperfine interaction are of the same order of magnitude. The numerical values depend strongly on what we called the six-quark bag probability. This is the probability that two-nucleons make a 
close enough encounter so that it is correct that the quark degrees of freedom can be used. We had some a sensible prescription [59], but unfortunately there is not yet any convincing evidence that this probability is non-vanishing. This affirms what everyone knows: the quark presence in nuclei is difficult to establish. It is hard to separate quark effects from those obtained from other mechanisms.

The work of Ref. [57] initiates a model of nucleon-nucleon scattering that is consistent with scattering lengths. This is then used to compute the $A=3$ binding energy difference, using a wave function that is expressed in terms of nucleon coordinates However, it is well known that using any potential that accounts for the scattering length difference will account for the $A=3$ binding energy difference. Thus the computed agreement for $A=3$ provided in this reference provides no (nada, nil, rien de tout) evidence for the particular Coulomb mechanism used to create the potential. Furthermore, the claim of [57] that the Coulomb interaction is exclusively responsible for the nucleon-nucleon scattering length differences is incomplete. There are other mechanisms that are non-zero. Most probably, their underlying non-relativistic quark model is not sufficiently accurate for the problem at hand. The claims in the abstract are not supported by sufficient evidence.

In my opinion, modern tools such as lattice gauge theory calculations will eventually settle the issue. It has already been calculated [60] that the influence of up-down quark mass difference on the $n-p$ mass difference is about 2.5 times larger in magnitude than that of electromagnetic effects.

\section{CONCLUDING REMARKS}

Each of the previous sections ends with some sort of a conclusion, so I'd like to wrap up by making further remarks about Ernest Henley. Ernest's isospin vision, laid out in his reviews, was carried out by a host of experimentalists and theorists. This program was carried out with great success and much was learned. It is perhaps more important to recognize that Ernest always acted with grace in difficult situations. His actions provided a model of the correct way to behave. I have tried to emulate his behavior. I am very grateful for having known him and will miss him as long as it is possible.

\section{Acknowledgements}

This work was supported by the U. S. Department of Energy Office of Science, Office of Nuclear Physics under Award Number DE-FG02-97ER-41014.

[1] E. H. Henley, "Charge independence and charge symmetry of nuclear forces" in "Isospin in Nuclear Physics," edited by D. H.Wilkinson, North Holland, Amsterdam, (1970)

[2] W. R. Gibbs, B. F. Gibson and G. J. Stephenson, Phys. Rev. C 11, 90 (1975) Erratum: [Phys. Rev. C 12, 2130 (1975)].

[3] B. Gabioud et al., Phys. Rev. Lett. 42, 1508 (1979).

[4] Q. Chen et al., Phys. Rev. C 77, 054002 (2008).

[5] E. M. Henley and G. A. Miller, in Mesons In Nuclei, Vol.I, Edited by M. Rho, and D. Wilkinson, North Holland, Amsterdam 1979, p. 405.

[6] C. Y. Cheung, E. M. Henley and G. A. Miller, Nucl. Phys. A 305, 342 (1978).

[7] M. A. Alberg, E. M. Henley, G. A. Miller and J. F. Walker, Nucl. Phys. A 306, 447 (1978).

[8] C. Y. Cheung, E. M. Henley and G. A. Miller, Nucl. Phys. A 348, 365 (1980).

[9] G. A. Miller, B. M. K. Nefkens and I. Slaus, Phys. Rept. 194, 1 (1990).

[10] G. A. Miller and W. T. H. Van Oers, In Symmetries and fundamental interactions in nuclei, Edited by W. C. Haxton, and E. M. Henley World Scientic, Singapore 1995, p. 127.

[11] G. A. Miller, A. K. Opper and E. J. Stephenson, Ann. Rev. Nucl. Part. Sci. 56, 253 (2006)

[12] R. Abegg, D. Bandyopadhyay, J. Birchall, E. W. Cairns, H. Coombes, C. A. Davis, N. E. Davison and P. P. J. Delheij et al., Phys. Rev. Lett. 56, 2571 (1986). 
[13] R. Abegg, D. Bandyopadhyay, J. Birchall, E. B. Cairns, G. H. Coombes, C. A. Davis, N. E. Davison and P. P. J. Delheij et al., Phys. Rev. D 39, 2464 (1989).

[14] R. Abegg, A. R. Berdoz, J. Birchall, J. R. Campbell, C. A. Davis, P. P. J. Delheij, L. Gan and P. W. Green et al., Phys. Rev. Lett. 75, 1711 (1995).

[15] J. Zhao, R. Abegg, A. R. Berdoz, J. Birchall, J. R. Campbell, C. A. Davis, P. P. J. Delheij and L. Gan et al., Phys. Rev. C 57, 2126 (1998).

[16] L. D. Knutson, S. E. Vigdor, W. W. Jacobs, J. Sowinski, P. L. Jolivette, S. W. Wissink, C. Bloch and C. Whiddon et al., Phys. Rev. Lett. 66, 1410 (1991).

[17] S. E. Vigdor, W. W. Jacobs, L. D. Knutson, J. Sowinski, C. Bloch, P. L. Jolivette, S. W. Wissink and R. C. Byrd et al., Phys. Rev. C 46, 410 (1992).

[18] R. B. Wiringa, S. Pastore, S. C. Pieper and G. A. Miller, Phys. Rev. C 88, 044333 (2013).

[19] G. A. Miller, A. W. Thomas and A. G. Williams, Phys. Rev. Lett. 56, 2567 (1986).

[20] A. G. Williams, A. W. Thomas and G. A. Miller, Phys. Rev. C 36, 1956 (1987).

[21] E. J. Stephenson, A. D. Bacher, C. E. Allgower, A. Gardestig, C. Lavelle, G. A. Miller, H. Nann and J. Olmsted et al., Phys. Rev. Lett. 91, 142302 (2003).

[22] A. Gardestig, C. J. Horowitz, A. Nogga, A. C. Fonseca, C. Hanhart, G. A. Miller, J. A. Niskanen and U. van Kolck, Phys. Rev. C 69, 044606 (2004)

[23] A. Nogga, A. C. Fonseca, A. Gardestig, C. Hanhart, C. J. Horowitz, G. A. Miller, J. A. Niskanen and U. van Kolck, Phys. Lett. B 639, 465 (2006)

[24] T. A. Lahde and G. A. Miller, Phys. Rev. C 75, 055204 (2007)

[25] A. C. Fonseca, R. Machleidt and G. A. Miller, Phys. Rev. C 80, 027001 (2009)

[26] P. Adlarson et al. [WASA-at-COSY Collaboration], Phys. Lett. B 739, 44 (2014)

27] P. Adlarson et al. [WASA-at-COSY Collaboration], Phys. Lett. B 781, 645 (2018)

[28] A. K. Opper, E. J. Korkmaz, D. A. Hutcheon, R. Abegg, C. A. Davis, R. W. Finlay, P. W. Green and L. G. Greeniaus et al., Phys. Rev. Lett. 91, 212302 (2003).

[29] S. Weinberg, Physica A 96, no. 1-2, 327 (1979).

[30] U. van Kolck, Prog. Part. Nucl. Phys. 43, 337 (1999)

[31] P. F. Bedaque and U. van Kolck, Ann. Rev. Nucl. Part. Sci. 52, 339 (2002)

[32] C. Hanhart, Phys. Rept. 397, 155 (2004)

[33] S. Weinberg, Lect. Notes Phys. 452, 1 (1995)

[34] van Kolck U. Few-Body Syst. Suppl. 9:444 (1995); U. of Texas Ph.D. Dissertation (1993)

[35] U. van Kolck, J. L. Friar and J. T. Goldman, Phys. Lett. B 371, 169 (1996).

[36] D. S. Armstrong and R. D. McKeown, Ann. Rev. Nucl. Part. Sci. 62, 337 (2012)

[37] D. Androic et al. [Qweak Collaboration], arXiv:1307.5275 [nucl-ex].

[38] D. Androic et al. [Qweak Collaboration], Nature 557, no. 7704, 207 (2018).

[39] V. Dmitrasinovic and S. J. Pollock, Phys. Rev. C 52, 1061 (1995)

[40] G. A. Miller, Phys. Rev. C 57, 1492 (1998)

[41] B. Kubis and R. Lewis, Phys. Rev. C 74, 015204 (2006).

[42] H. H. Matevosyan, A. W. Thomas and G. A. Miller, Phys. Rev. C 72, 065204 (2005)

[43] H. H. Matevosyan, G. A. Miller and A. W. Thomas, Phys. Rev. C 71, 055204 (2005)

[44] P. C. McNamee, M. D. Scadron and S. A. Coon, Nucl. Phys. A 249, 483 (1975).

[45] S. A. Coon and R. C. Barrett, Phys. Rev. C 36, 2189 (1987).

[46] P. G. Blunden and M. J. Iqbal, Phys. Lett. B 198, 14 (1987).

[47] M. Wagman and G. A. Miller, Phys. Rev. C 89, no. 6, 065206 (2014) Erratum: [Phys. Rev. C 91, no. 1, 019903 (2015)]

[48] G. A. Miller and A. Schwenk, Phys. Rev. C 78, 035501 (2008).

[49] G. A. Miller and A. Schwenk, Phys. Rev. C 80, 064319 (2009).

[50] G. P. Zeller et al. [NuTeV Collaboration], Phys. Rev. Lett. 88, 091802 (2002) Erratum: [Phys. Rev. Lett. 90, 239902 (2003)]

[51] E. A. Paschos and L. Wolfenstein, Phys. Rev. D7, 91, (1973).

[52] E. Sather, Phys. Lett. B 274, 433 (1992).

[53] E. N. Rodionov, A. W. Thomas and J. T. Londergan, Mod. Phys. Lett. A 9, 1799 (1994).

[54] J. T. Londergan and A. W. Thomas, Phys. Lett. B 558, 132 (2003)

[55] J. T. Londergan and A. W. Thomas, Phys. Rev. D 67, 111901 (2003)

[56] G. A. Miller and A. W. Thomas, Int. J. Mod. Phys. A 20, 95 (2005)

[57] W. R. Gibbs and J. P. Dedonder, Phys. Rev. C 96, 034001 (2017)

[58] V. Koch and G. A. Miller, Phys. Rev. C 31, 602 (1985) Erratum: [Phys. Rev. C 32, 1106 (1985)].

[59] E. M. Henley, L. S. Kisslinger and G. A. Miller, Phys. Rev. C 28, 1277 (1983).

[60] S. Borsanyi et al., Science 347, 1452 (2015) 